\title{
Development-dependent changes in the tight DNA-protein complexes of barley on chromosome and gene level
} Tatjana Sjakste*1, Kristina Bielskiene ${ }^{2}$, Marion Röder ${ }^{3}$, Olga Sugoka1, Danute Labeikyte ${ }^{2}$, Lida Bagdoniene ${ }^{2}$, Benediktas Juodka², Yegor Vassetzky ${ }^{4}$ and Nikolajs Sjakste 5

\author{
Address: ${ }^{1}$ Genomics and Bioinformatics, Institute of Biology, University of Latvia, Miera 3, LV2169 Salaspils, Latvia, ${ }^{2}$ Department of Biochemistry \\ and Biophysics, Vilnius University, M. K. Čiurlionio 21, LT2009 Vilnius, Lithuania, ${ }^{3}$ Gene and Genome Mapping, Leibniz Institute of Plant \\ Genetics and Crop Plant Research, Correnstrasse 3, 06466, Gatersleben, Germany, ${ }^{4}$ UMR-8126, Institut Gustave Roussy, 39, rue Camille- \\ Desmoulins, 94805 Villejuif, France and 5Faculty of Medicine, University of Latvia, Šarlotes 1a, LV1001, Riga, Latvia \\ Email: Tatjana Sjakste* - tanja@email.lubi.edu.lv; Kristina Bielskiene - kristina.bielskiene@gf.stud.vu.lt; Marion Röder - roder@ipk- \\ gatersleben.de; Olga Sugoka - olga@email.lubi.edu.lv; Danute Labeikyte - Danute.Labeikyte@gf.vu.lt; \\ Lida Bagdoniene - Lida.Bagdoniene@gf.vu.lt; Benediktas Juodka - benediktas.juodka@cr.vu.lt; Yegor Vassetzky - vassetzky@igr.fr; \\ Nikolajs Sjakste - Nikolajs.Sjakste@lu.lv \\ * Corresponding author
}

Published: 12 May 2009

BMC Plant Biology 2009, 9:56 doi:10.1 186/147/-2229-9-56
Received: 23 December 2008

Accepted: 12 May 2009

This article is available from: http://www.biomedcentral.com/I47/-2229/9/56

(c) 2009 Sjakste et al; licensee BioMed Central Ltd.

This is an Open Access article distributed under the terms of the Creative Commons Attribution License (http://creativecommons.org/licenses/by/2.0), which permits unrestricted use, distribution, and reproduction in any medium, provided the original work is properly cited.

\begin{abstract}
Background: The tightly bound to DNA proteins (TBPs) is a protein group that remains attached to DNA with covalent or non-covalent bonds after its deproteinisation. The functional role of this group is as yet not completely understood. The main goal of this study was to evaluate tissue specific changes in the TBP distribution in barley genes and chromosomes in different phases of shoot and seed development. We have: I. investigated the TBP distribution along Amy $32 b$ and Bmyl genes encoding low pl $\alpha$-amylase $A$ and endosperm specific $\beta$ amylase correspondingly using oligonucleotide DNA arrays; 2 . characterized the polypeptide spectrum of TBP and proteins with affinity to TBP-associated DNA; 3 . localized the distribution of DNA complexes with TBP (TBPDNA) on barley $\mathrm{IH}$ and $7 \mathrm{H}$ chromosomes using mapped markers; 4. compared the chromosomal distribution of TBP-DNA complexes to the distribution of the nuclear matrix attachment sites.

Results: In the Amy32b gene transition from watery ripe to the milky ripeness stage of seed development was followed by the decrease of TBP binding along the whole gene, especially in the promoter region and intron II. Expression of the Bmy I gene coupled to ripening was followed by release of the exon III and intron III sequences from complexes with TBPs. Marker analysis revealed changes in the association of chromosome IH and $7 \mathrm{H}$ sites with TBPs between first leaf and coleoptile and at Zadoks 07 and Zadoks 10 stages of barley shoot development. Tight DNA-protein complexes of the nuclear matrix and those detected by NPC-chromatography were revealed as also involved in tissue- and development-dependent transitions, however, in sites different from TBP-DNA interactions. The spectrum of TBPs appeared to be organ and developmental-stage specific. Development of the first leaf and root system (from Zadoks 07 to Zadoks 10 stage) was shown as followed by a drastic increase in the TBP number in contrast to coleoptile, where the TBPs spectrum became poor during senescence. It was demonstrated that a nuclear protein of low molecular weight similar to the described TBPs possessed a high affinity to the DNA involved in TBP-DNA complexes.
\end{abstract}

Conclusion: Plant development is followed by redistribution of TBP along individual genes and chromosomes. 


\section{Background}

Reorganization of the chromatin structure is one of the main mechanisms for regulation of gene expression in plants, chromatin rearrangements take place in response to light and tissue-specific signalling molecules [1]. Despite rapid progress in the field, the functional significance of some groups of nuclear proteins, including the tightly bound to DNA proteins (TBP), remains obscure. TBP is a specific nuclear protein group that remains attached to DNA with covalent or non-covalent bonds after its deproteinisation independently of the deproteinisation method applied: protease digestion, phenol extraction, chloroform extraction or salting-out. The TBP have been found in the DNA of numerous evolutionary distant species [2]. Both the function of the TBPs and the nature of DNA sequences involved in the tight complexes remain to be detailed. Enrichment of the TBPs in specific DNA sequences is of special interest in connection with speculation on the potential function of such sequences in higher order structures of the genome of different organisms including humans, mouse, and chicken [3-6]. In our recent work [7] we have applied the DNA microarray technique to study the distribution of TBPs along the chicken alpha-globin domain in cell lines that expressed the gene, did not express it or conducted abortive expression. In this study we have shown profound transcriptiondependent changes in the TBP-distribution pattern in the alpha-globin domain. Other preliminary results from of our team [8] also indicated the existence of tissue- and development specificity in the patterns of TBP distribution in barley (Hordeum vulgare) shoots.

The life cycle of the barley plant, especially the shoot and seed provide an excellent model for plant development studies. Barley shoot and seed developmental stages are well characterized and classified [9]. Etiolated barley seedlings provide cell populations with different proliferation, differentiation and senescence status including synchronously dividing cell populations of primary leaf [10-12], senescent coleoptiles and a mixed cell population from roots [10]. In the present work we used dry grains (Zadoks 0 ) and grains after $20 \mathrm{~h}$ of imbibition (Zadoks 1); coleoptiles, first leaves and roots were dissected from shoots of Zadoks 07 (coleoptile emerged stage, classification according to [9]) and Zadoks 10 (first leaf through coleoptile) development stages as well as seeds of watery ripe (Zadoks 71) and medium milk development (Zadoks 75).

Moreover, expression of several specific enzymes of carbohydrate metabolism in barley is development- or/and tissue specific and is restricted to well-defined stages of the plant development [13]. It was reported that transcription of $\alpha$-amylase genes is low and decreases during seed development, but $\beta$-amylase expression in endosperm is upregulated during its development [13-15]. Two represent- atives of each group of amylase genes, the $A m y 32 b$ and Bmy1 genes provide prospective model systems. Highly conserved in barley cultivars, Amy32b gene [16] is located on chromosome $7 \mathrm{H}$ in the centromere region [17]. Gene encodes a low-pI $\alpha$-amylase and is expressed in barley aleurone cells under the control of gibberellic acid and abscisic acid [15]. Highly polymorphic in structure, barley Bmy1 gene $[18,19]$ is located in the long arm of chromosome $4 \mathrm{H}[20]$, encodes endosperm specific $\beta$-amylase and is expressed only during seed development [18]. Several allelic forms of Bmy1 structural gene were sequenced and analyzed in relation to their functionality [21-24].

Despite the fact that barley genome is not completely sequenced, work with this plant provides the opportunity to use the technique of mapped barley genomic markers developed during recent decades $[25,26]$. The tool allows chromosome profiling to be performed in any application.

In order to study transcription, tissue and development dependent changes in TBPs distribution in barley genes and chromosomes at different phases of shoot and seed development, we have formulated the following as the goals of the present study: 1 . to investigate the distribution of TBPs along Amy32b and Bmy1 genes using oligonucleotide DNA arrays; 2 . to characterize the polypeptide spectrum of TBP in different shoot organs and during different development stages. 3. to apply mapped barley MS as a tool to study tissue and developmental specificity in the distribution of DNA complexes with TBP (TBP-DNA) in the barley chromosomes $1 \mathrm{H}$ and $7 \mathrm{H} ; 4$. to compare the chromosomal distribution of TBP-DNA complexes and distribution of tight DNA-protein complexes separated using other than TBP isolation approaches including nuclear matrix isolation and nucleoprotein chromatography on celite (NPC-chromatography).

\section{Results \\ RT-PCR}

Figure 1 presents data on $A m y 32 b$ (lanes 1, 2) and Bmy1 (lanes 3,4) genes expression in seeds of watery ripe (Zadoks 71, lanes 1, 3) and medium milk (Zadoks 75, lanes 2,4) stages of development. Reference gene Tub1, a ubiquitous and stably expressed gene in barley, was expressed with the same intensity at both stages analyzed. It was found that $A m y 32 b$ gene is highly expressed in watery ripe and silent in milky ripe seeds. On the contrary, expression of the Bmy1 gene was detected exclusively in the milky ripe stage. Thus, the chosen barley genes at two stages of seed development represented a model system to be used in further analysis of TBPs distribution along the silent and expressed genes. 


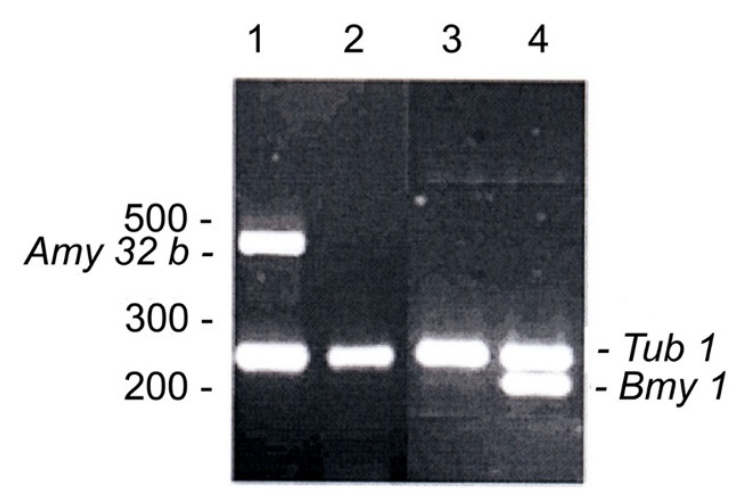

Figure I

Amy32b (lanes I, 2), Bmy I (lane 3, 4) and alpha tubulin (lanes I - 4) RT-PCR products. Lanes I, 3 - RNA from watery ripe seeds; lanes 2, 4 - RNA from milky ripe barley seeds. Positions of the molecular weight markers (bp) are indicated on the right. Positions of RT-PCR products are indicated on both sides of the figure.

\section{Microarray analysis}

Figure 2 and Figure 3 present quantified results of hybridization between TBP-DNA complexes fractionated from seeds of watery ripe (Zadoks 71, panels A) and medium milk (Zadoks 75, panels B) stages and DNA microarray of Amy32b (Figure 2) and Bmy 1 (Figure 3) genes correspondingly. In samples from watery ripe seeds, ratios of hybridization intensities with $\mathrm{R}$ probe (probe derived from the DNA complexed with tightly bound proteins) and $\mathrm{F}$ probe (probe derived from the TBP-free DNA) and $A m y 32 b$ microarray were approximately the same in oligonucleotide positions 1, 9, 10 and 11 reflecting an equal or similar amount of the corresponding DNA fragments in both TBP bound and TBP unbound fractions. In all other positions the ratio exceeded 1, indicating the enrichment in TBPs in the corresponding DNA fragments. Positions 3 (300 bp upstream the translation start codon), 7 (downstream part of Intron 2), and 8 (Exon 3 ) were shown as the most TBP-enriched in samples from watery-ripe seeds. Transition to milky ripening was followed by the decrease of $\mathrm{R}$ vs $\mathrm{F}$ ratio in general along the whole $A m y 32 b$ gene. Ratio much below 1 in positions 1, 5,9 and 11 indicates predominant accumulation of corresponding DNA fragments in the TBP- free $\mathrm{F}$ fraction. In positions 3 and 7 the ratio decreased from 6 and 4 to almost 1, and from 4 to 2.5 in position 8 . The position of oligonuclotide 10 , in which the ratio increased from 1 to 2 , was an exception. Thus, during seed development, an overall decrease in TBP-DNA interactions ( $\mathrm{R} \rightarrow \mathrm{F}$ transition) occurs along the
Amy32b gene being most drastic in the promoter and Intron 2 gene regions.

The $\mathrm{R}$ and $\mathrm{F}$ fractions obtained from watery ripe seeds hybridized also with different intensity with $B m y 1$ gene microarray. An R vs F ratio close to or less than 1 was observed for positions 3 and 11 , and 4 and 12 correspondingly. In positions 1, 2, 5, and 8 hybridization with $\mathrm{R}$ probes was somewhat stronger than with $\mathrm{F}$ probes, however the $\mathrm{R}$ vs $\mathrm{F}$ ratio did not exceed 2 . The $\mathrm{R}$ vs $\mathrm{F}$ ratio was rather high in oligonucleotide positions 6, 9 (more than 2 ) and 13 (near to 3). Finally, in positions 7 and 10, the intensity of hybridization with $\mathrm{R}$ probe was 4 times stronger than with F probe. The highest ratio in the milky ripeness stage (Figure 3, panel B) was close to 2 in position 10, and slightly exceeded 2 in position 13 . In all other oligonucleotide positions the $\mathrm{R}$ vs $\mathrm{F}$ ratio was around 1 . Thus, similar to the results obtained with Amy32b gene, the overall decrease of the $\mathrm{R} v \mathrm{~F}$ ratio is revealed along the $B m y 1$ gene during seed development. The process is more pronounced in Exon 3 and upstream the microsatellite locus in Intron III.

\section{Bioinformatic analysis of Amy32b and Bmy I gene sequences}

Identification of specific areas of TBP binding in both genes raised the question of peculiarities in the gene sequences in these areas. A search for nuclear matrix attachment regions (MARs) performed by the MatInspector program (Rel. 7.4), revealed a possible MAR in Intron 3 of the Bmy1 gene (Figure 3). No MARs were detected in the Amy32b gene. DiAlign TF, Release 3.1 revealed 13 homology regions between the two genes (Figures 2 and 3 ), however no common features in the distribution of transcription factor binding sites along the genes and transcription factor binding modules were revealed. Comparison of the predicted MAR with the TBP binding sites in the Bmy1 gene Intron 3 indicates that this site is enriched in TBPs. Similar sequences of the two genes differed in their affinity to TBPs. The only exception was observed in the area of similarity S9; these sites were TBP-enriched in both genes.

\section{Profiling of DNA-protein complexes along chromosomes}

Positive data on intragenic changes in TBP distribution in the course of development encouraged us to upscale the investigation and to study the long-range distribution of tightly bound proteins along barley chromosomes $1 \mathrm{H}$ and $7 \mathrm{H}$. Lack of barley genome sequence information makes it impossible to apply microarray technology to study long-range distribution of TBPs along chromosomes. To at least partly reach the goal we have used the tool of mapped markers well developed in barley studies [25-27]. Profiling experiments were designed as PCRbased identification of the DNA matrices in DNA-protein 

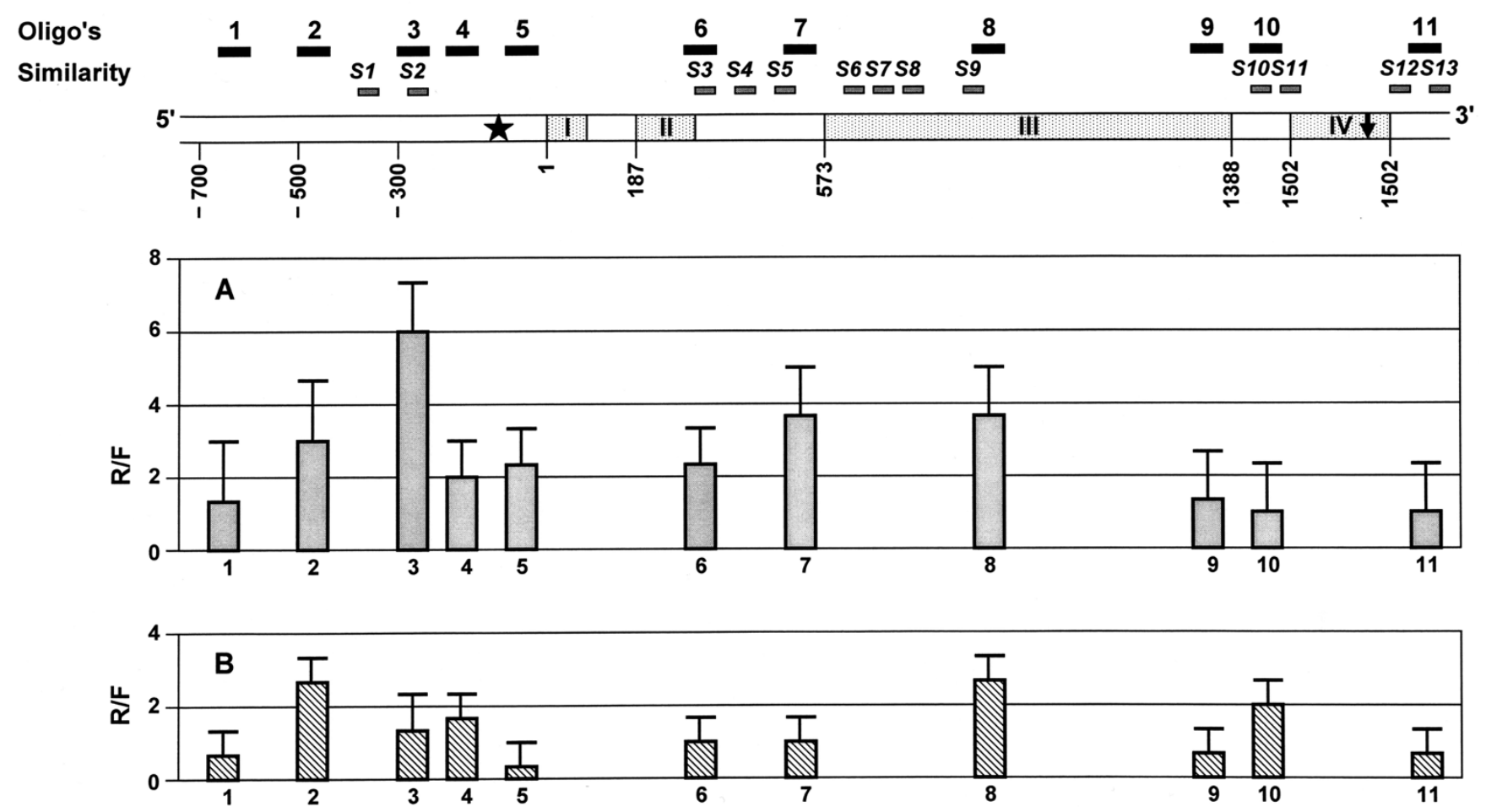

Figure 2

DNA array based mapping of the TBPs distribution in Amy32b structural gene in watery ripe (A) and milky ripe (B) barley seeds. Upper panel presents the gene structure with positions of oligonucleotides of the array and regions of the similarity with Bmyl (SI - SI3). Star indicates position of the TATA box, black arrow in last exon indicates stop codon. Exon numbers are given as Roman numerals. The data in lower panels represent the ratio of hybridization of R vs F DNA fractions scored as an average of three independent experiments (two hybridizations per experiment). Error bars represent standard deviation.

complexes obtained by different fractionation approaches. Involvement of the DNA stretch in interactions with a given protein group (TBP, nuclear matrix) was scored in terms of the presence/absence of amplification with mapped markers of barley chromosomes $1 \mathrm{H}$ and 7 H. Data are organized as graphical representations of chromosome $1 \mathrm{H}$ and $7 \mathrm{H}$ with an indication of the PCR result at each MS locus in all DNA-protein fractions analyzed. The study was performed on DNA from barley shoot organs; this model enables us to obtain a sufficient quantity of material for fractionation of nuclear structures.

\section{TBPs}

Figure 4 presents graphically both chromosomes as profiles of TBP-DNA complexes in seeds before (Zadoks 0) and after imbibition (Zadoks 1), as well as leaves and coleoptiles at two stages of development (Zadoks 07 and 10). In dry seeds most markers of both chromosomes were found in F and R1 fractions. Only four and five markers of $1 \mathrm{H}$ and $7 \mathrm{H}$ correspondingly were present in the R2 fraction. Three markers of 1H (GBMS0065, Bmac0154,
Bmag0718) were found exclusively in the filtered TBP-free DNA (Figure 4A). However, positive amplification in R1 was detected in the above loci after imbibitions. Positive amplification in the R2 fraction with Bmac0090, HVHVA1, and EBmac0783 of $1 \mathrm{H}$ and with Bmag0914 of $7 \mathrm{H}$ was also obtained only after imbibition. These results indicate an increase in the number of the tight interaction sites between TBPs and DNA. In contrast, the loci Bmag0382, GBMS0184 and WMCIE8 of $1 \mathrm{H}$ and AF022725A, cMWG 728 and GBMS0183 of 7H disappeared from the R2 fraction during transition from the Zadoks 0 to the Zadoks 1 stage. In stage 07 leaves some regions of both chromosomes appear to be free of TBPs. No markers of chromosome $1 \mathrm{H}$ and only two $7 \mathrm{H}$ markers were found in $\mathrm{R} 2$ in Zadoks 07 leaves. In Zadoks 07 leaves in largest number of loci interacting with TBPs was decreased when compared to dry seeds. Only two loci on Chromosome 1H (Bmac0154 and Bmag0718), formerly unbound to TBP became involved in complexes with TBP. Markers Bmac0187 and ABC 156 D of 7H appeared in R2 fraction and disappeared from $\mathrm{F}$ fraction correspondingly. The transition of the leaf to the Zadoks10 stage was fol- 

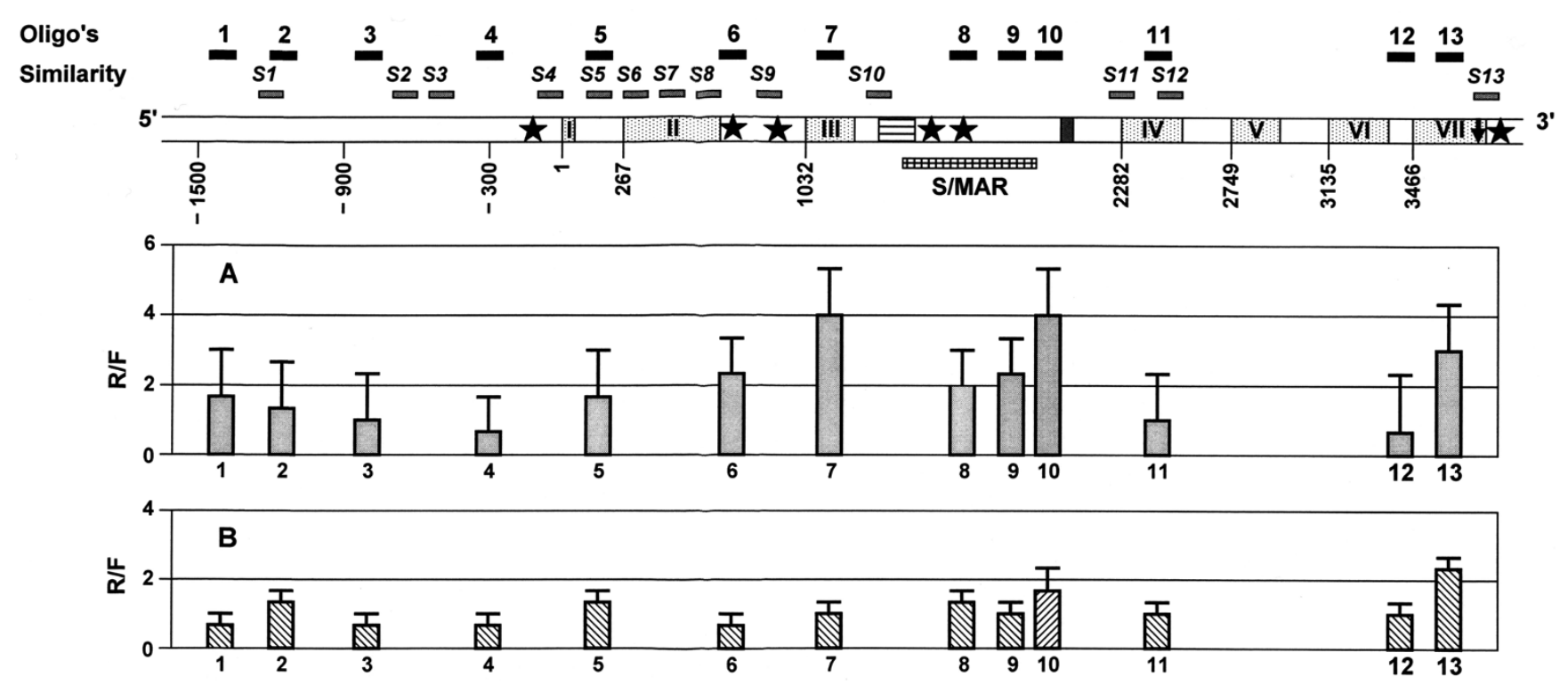

Figure 3

DNA array based mapping of the TBPs distribution in Bmyl structural gene in watery ripe (A) and milky ripe (B) barley seeds. Upper panel presents the gene structure with positions of oligonucleotides of the array and regions of similarity with Amy32b gene (SI - SI3). Stars correspond to the positions of the TATA boxes; black arrow in last exon VII indicates the stop codon. Exon numbers are given in Roman numerals. Black and dashed squares in the Intron III indicate the positions of the microsatellite and MITE element, correspondingly. The predicted MAR position is indicated by a checked bar. The data on lower panels represent the ratio of hybridization of R vs F DNA fractions scored as an average of three independent experiments (two hybridizations per experiment). Error bars represent standard deviation.

lowed by a substantial increase in stretches involved in complexes with TBPs when compared to Zadoks 07 stage (positive amplification appeared in R1 fractions at most loci). However, DNA templates were not detected in R2 fractions along both chromosomes except for the GBMS0012 and GBMS0184 loci of 1H. Interestingly, in stage 07 coleoptiles the number of sites involved in TBPDNA interactions was somewhat greater than in dry seeds (most markers were found in the R1 fraction, several also in the $\mathrm{R} 2$ fraction). The transition to stage 10 in coleoptiles was followed a decrease in the number of genomic sites involved in interaction with TBPs. In the stage 10 coleoptiles only one marker of $1 \mathrm{H}$ and two markers of $7 \mathrm{H}$ were found in the R2 fraction, some markers disappeared from the R1 fraction.

Panel B in Figure 4 summarizes the trends of transitions during development of different organs compared with the situation in dry seed. Panel $\mathrm{C}$ gives a summary of the involvement of the Chromosome $1 \mathrm{H}$ and $7 \mathrm{H}$ sites of the $\mathrm{R} \leftrightarrow \mathrm{F}$ transitions. It emerges that all the sites on chromosome $7 \mathrm{H}$ are involved, and only four sites of $1 \mathrm{H}$ are not involved in the dynamics. Thus, the development of the barley seedling is coupled to rearrangements in the interactions of DNA with TBPs both on chromosome $1 \mathrm{H}$ and $7 \mathrm{H}$. The process is organ specific and can be termed "tight- ening" of the DNA-TBP interactions in the leaves and "loosening" in the coleoptile compared to dry seed status.

\section{Chromatin fractions and nuclear matrix}

Figure 5 presents the distribution of molecular markers in DNS fragments in soluble chromatin (SC), insoluble chromatin (IC) and nuclear matrix (NM) fractions extracted from leaves at the Zadoks 07 and Zadoks 10 stages. In stage 07 leaves, the markers EBmac0560, GBMS0065, Bmag0718, GBMS0143, GBMS0012, WMCIE8 of $1 \mathrm{H}$ and markers cMWG773, Bmag0516, cMWG725, EBmac0755, ABG461 of 7H were found in the nuclear matrix-attached DNA. All these markers moved to the insoluble chromatin fraction at stage 10 . Thus senescence of the first leaf appears to be accompanied by a "loosening" of the DNA-NM interactions accompanied by complete detachment of some chromosomal regions of the nuclear matrix. The right panel of the Figure 5 indicates the sites involved in transitions in DNA-chromatin and DNA-NM interactions. Interestingly, data obtained on both chromosomes indicate that in our model many fewer chromosomal sites appear to be involved in the transitions of DNA -NM interactions compared to the number of $\mathrm{R} \leftrightarrow \mathrm{F}$ transition loci revealed by the profiling of the TBP-DNA complexes (Figure 4A, D; Figure 5). 
$1 \mathrm{H}$

\section{A}

\begin{tabular}{|c|c|c|c|c|c|c|}
\hline Zadoks stage & 0 & 1 & 7-L & 10-L & 7-C & $10-c$ \\
\hline TBP fraction & 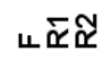 & 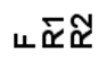 & 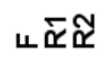 & 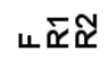 & 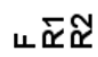 & u $\bar{\Upsilon} \tilde{\Upsilon}$ \\
\hline
\end{tabular}

Marker $\mathbf{C m}$

Bmac0213 28

HvALAAT 57 Cen

Bmac0090 58 37-44

EBmac0560 58

EBmac0656 63

GBMS0065 66

EBmac0659 67

GBMS0037 71

Bmac0154 81

Bmag0382 97

Bmag0718 112

HVHVA1 112

GBMS0053 132

GBMS0012 136

GBMS0143 145

GBMS0184 150

WMCIE8 164

Bmag0579 175

EBmac0783 180
PCR results

B

Dynamics in TBP

o- $-\frac{1}{0}$ 잉
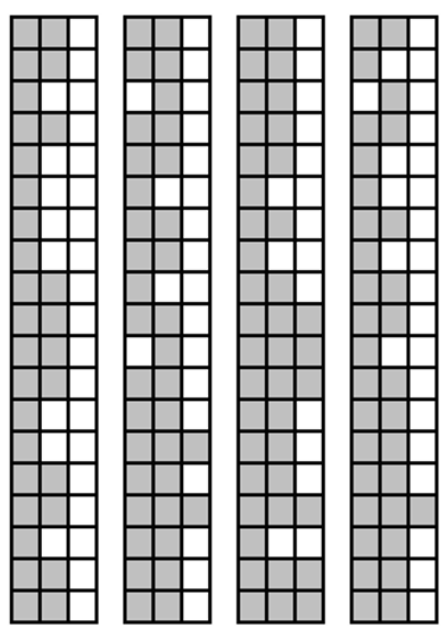

C

Involvement

in dynamics

$1 \mathrm{H}$
$7 \mathrm{H}$

A

\section{PCR results}

\section{Zadoks stage}

TBP fraction

$\begin{array}{lr}\text { Marker } & \text { Cm } \\ \text { GBMS0192 } & 4 \\ \text { Bmag0206 } & 19 \\ \text { GBMS0139 } & 25 \\ \text { Bmag0007 } & 27 \\ \text { GBMS0035 } & 49 \\ \text { EBmac0603 } & 50 \\ \text { AF022725A } & 59 \\ \text { Bmag0516 } & 73 \\ \text { Bmag0914 } & 73 \\ \text { Bmac0187 } & 89 \\ \text { ABC 156 D } & 93 \\ \text { GBMS0111 } & 100 \\ \text { Bmag0120 } & 118 \\ \text { cMWG 728 } & 158 \\ \text { GBMS0183 } & 161 \\ \text { GBM-128 }\end{array}$

Figure 4 (see legend on next page)
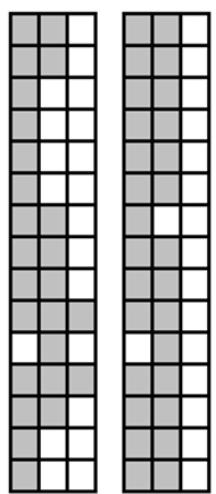

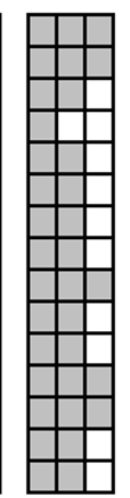
)
B

Dynamics in TBP

o-
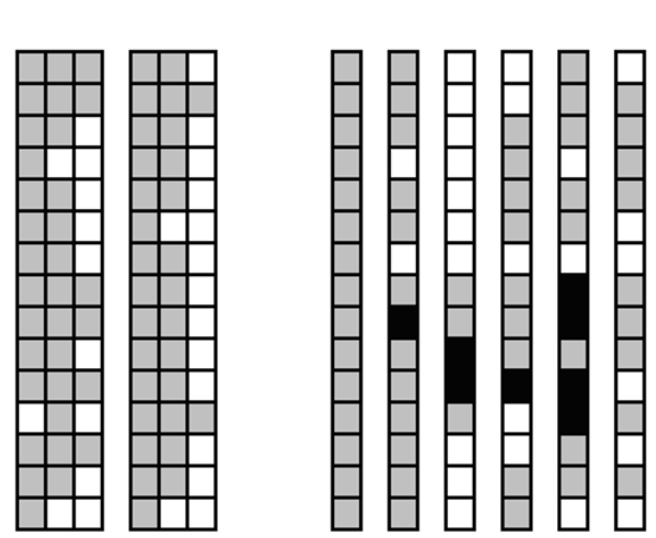

C

Involvement in dynamics

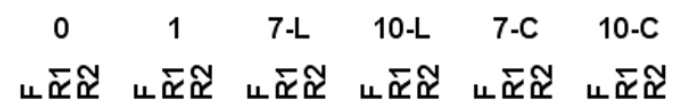


Figure 4 (see previous page)

The distribution of microsatellite sequences in free DNA and tight DNA-protein complexes along barley chromosomes $\mathbf{I H}$ and $7 \mathbf{H}$. A. The distribution of DNA fragments containing given microsatellites in free DNA (F), and tight DNA-protein complexes (RI and R2). Marker names are given in the left column, following column gives distance from the chromosome extremity in centimorganides. Position of the centromere is indicated separately (Cen). 0 - dry seeds (phase 0 ), I - 20 hours of imbibition (phase 0I); 7-L - first leaf on stage 07; I0-L - leaf on stage 10; 7-C - coleoptile on stage 07; I0-C coleoptile on stage 10. Grey squares - presence of amplification; white squares - absence of amplification. B. Summary of the trends of transitions during development of different organs compared with the situation in dry seed. Grey squares - situation in the seed or similar; white squares - decrease of association with TBPs; black squares - increase in TBP-DNA interactions. C. General summary of involvement of the studied genomic sites in R-F and adverse transitions. Black points on white background - site involved in transitions; white points on black background - site not involved in transitions.

\section{NPC chromatography}

The nucleoprotein chromatography method enables the discrimination of several types of DNA-protein interactions in the nucleus including two kinds of DNA complexes with nuclear matrix proteins that are not revealed by other approaches. In this method cell lysate is loaded onto a celite column. Celite irreversibly binds all proteins including protein moieties of the nucleoproteins. Nucleic acids are gradually released from the protein complexes by sequential gradients of $\mathrm{NaCl}$, LiCl-urea and temperature. DNA not bound to the nuclear matrix is eluted in a $\mathrm{NaCl}$ gradient (DNA 0), DNA "loosely" bound to the nuclear matrix is released using a LiCl-urea gradient (DNA I), high temperature is necessary to release DNA from the strong complexes with the nuclear matrix (DNA II). We suppose that single strand DNA breaks in the vicinity of the replication complex induce the transition DNAII DNA I, but double strand breaks release the DNA0 fraction $[28,29]$. Graphical presentations of chromosomes $1 \mathrm{H}$ and $7 \mathrm{H}$ (Figure 6) illustrate the results of amplification on the DNA purified from the different NPC-fractions: DNA 0, DNA I and DNA II $[28,29]$. In dry seeds all the $1 \mathrm{H}$ markers and most of the $7 \mathrm{H}$ markers were revealed in all three chromatographically analyzed fractions. Data obtained on both chromosomes, reflect complex patterns of chromatin domain reorganizations after imbibitions. Among 22 loci tested on $1 \mathrm{H}$, six disappeared from the DNA II fraction (Figure 6, 1H A and B). Among 24 markers used in $7 \mathrm{H}$ profiling, 11 became involved in looser interactions with the nuclear matrix (Figure 6, 7H, A and B). Development of the first leaf (Zadoks 07) was accompanied by an increase in loci number associated with the nuclear matrix including EBmac0560, Bmag0211, GBMS0065, Bmag0718, GBMS0143, WMCIE8 and HVPLASCIB, Bmag0007, GBMS0035, Bmag0507 for 1H and $7 \mathrm{H}$ correspondingly. Several loci became unbound to NM via the strong bond (GBMS0065, GBMS0012, GBMS0143 and ABG 461 for $1 \mathrm{H}$ and $7 \mathrm{H}$ correspondingly) at the Zadoks 10 stage. However, in general, senescence of the first leaf was followed by an increase in the sites interacting with the nuclear matrix (Figure 6, Panel B). Interestingly, in Zadoks10 coleoptiles the profiles of both chromosomes indicate detachment of DNA from nuclear matrix or loosening of the DNA-NM bonds in most loci tested. Only three (Bmac0213, Bmag0382, Bmag0579) and six (Bmag007, cMWG 773, GBMS0035, AF022725A, cMWG 725, ABG 461) loci from 22 and 24 tested correspondingly for $1 \mathrm{H}$ and $7 \mathrm{H}$ chromosomes remained tightly attached to the nuclear matrix. Most sites of both chromosomes were involved in the rearrangements with two exceptions for each chromosome. (Figure 6, Panel C).

\section{Tightly bound protein spectrum in organs of stage 07 and} stage 10 shoots

Figure 7 presents electropherograms of the TBPs obtained from the leaves, roots and coleoptiles of Zadoks stage 07 and Zadoks stage 10 shoots using method of exhaustive DNase digestion. Amazingly, the TBP spectrum appears to be organ and developmental stage-specific. In stage 07 leaves there is only one TBP of molecular weight $30 \mathrm{KDa}$, and the transition to stage 10 and senescence of the leaf is followed by a drastic increase of the TBP number, additional bands of 17, 21, 36, 42, 55, 60, 69 and $76 \mathrm{KDa}$ are clearly visible. And a similar process happens in the roots. Besides the $30 \mathrm{KDa}$ band a smaller $20 \mathrm{KDa}$ polypeptide is detected in young roots. Additional $36 \mathrm{KDa}$ protein and some minor high-molecular polypeptides are detectable in old roots. Changes in the TBP spectrum in coleoptile appear to be inversed. The TBP pattern in stage 07 coleoptile is rather complicated. There is a high molecular weight polypeptide of about $200 \mathrm{KDa}$, bands at 67 , and $38 \mathrm{KDa}$, as well as a $30 \mathrm{KDa}$ band common for all organs and a 20 KDa band found also in the roots. In contrast, only two polypeptides are found in stage 10 coleoptile, the $36 \mathrm{KDa}$ polypeptide, detected in all organs of stage 10 shoots and $20 \mathrm{KDa}$, also found in all organs, but it seems to be prominent in the coleoptile. Thus the polypeptide spectrum of TBPs appears to depend on the plant organ development stage. Further sets of experiments were performed to reveal specificity of interactions of TBPs with DNA sequences. 
$1 \mathrm{H}$

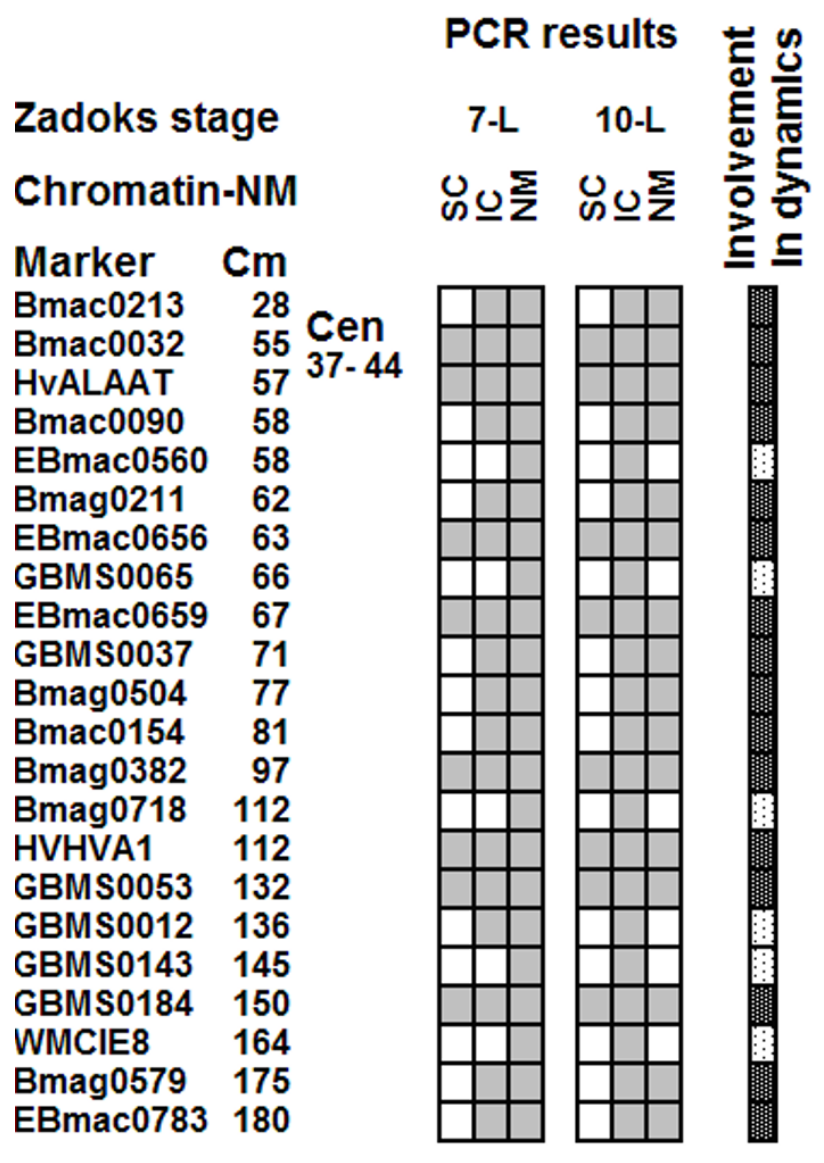

7H

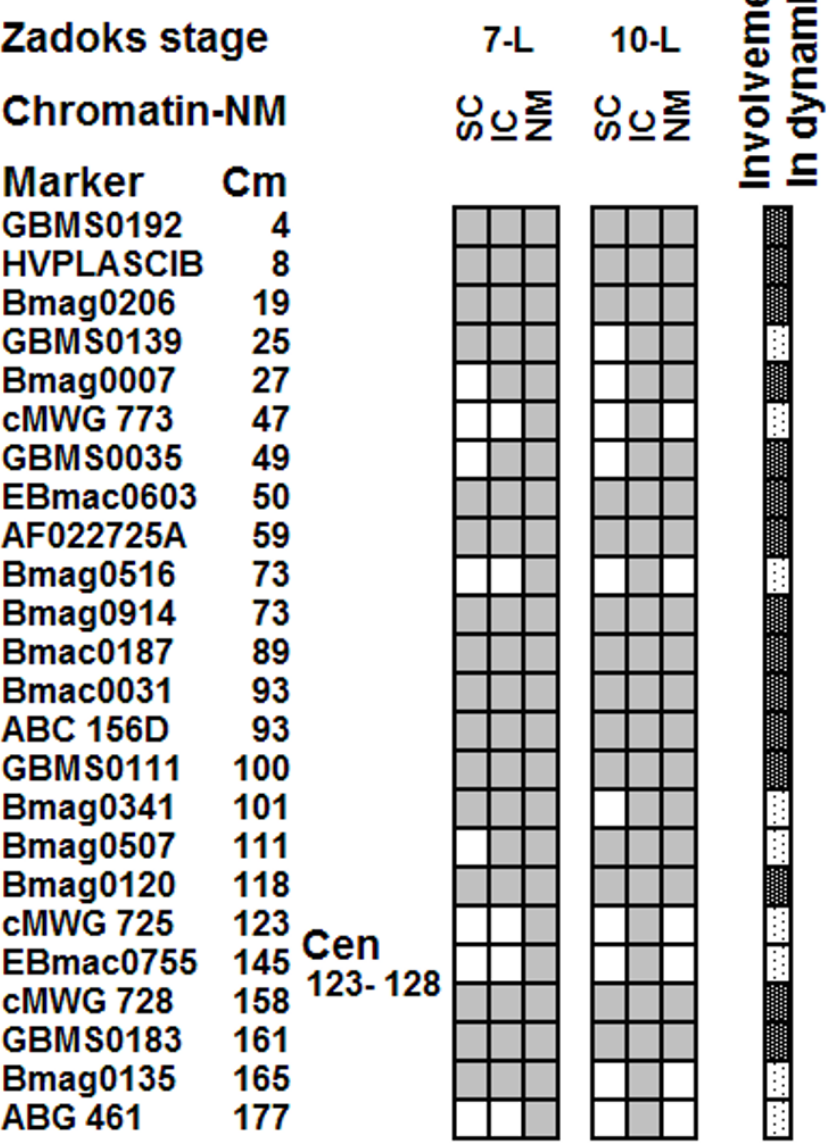

Figure 5

The distribution of microsatellite sequences in chromatin fractions and nuclear matrix-attached DNA along barley chromosomes $\mathbf{I H}$ and $\mathbf{7 H}$. Columns in the right part of the figure illustrate the distribution of DNA fragments containing given microsatellites in fractions of soluble chromatin (SC), insoluble chromatin (IC) and the nuclear matrix (NM). Panel on the right indicates involvement in association or dissociation of the nuclear matrix. Grey and white squares correspond to the presence and absence of amplification correspondingly. Black points on white background and white points on black background indicate on the sites involved and not involved in transitions. All other designations are as in Figure 4.

\section{Affinity of the TBP-bound DNA to nuclear proteins}

To test changes in the tight DNA-protein interactions coupled to plant development we performed DNA-binding protein blot assays with electrophoretically fractionated nuclear polypeptides extracted from different barley organs at development stages 07 and 10 and TBP-bound DNA from the stage 10 leaves. Results are shown in Figure 8. Nuclear polypeptides isolated from the stage 07 coleoptiles manifested high affinity to the TBP-associated DNA. Both low-molecular weight and $70 \mathrm{kDa}$ peptides formed tight complexes with DNA. Transition to stage 10 was associated with a decrease of DNA-binding proteins, only low-molecular weight proteins could bind to the probe
(Figure 8, lanes 1 and 4). Mostly low molecular weight polypetides extracted from stage 07 leaves manifested affinity to the probe, in stage 10 leaves these were replaced by $70 \mathrm{kDa}$ peptides (Figure 8, lanes 2 and 5). The TBPassociated DNA probe recognized low molecular nuclear polypeptides extracted from the stage 07 roots, in extracts from stage 10 roots binding to these polypeptides became less intense, however additional $35 \mathrm{kDa}$ and $70 \mathrm{kDa}$ polypeptides with affinity to the probe were detected. 
$1 \mathrm{H}$

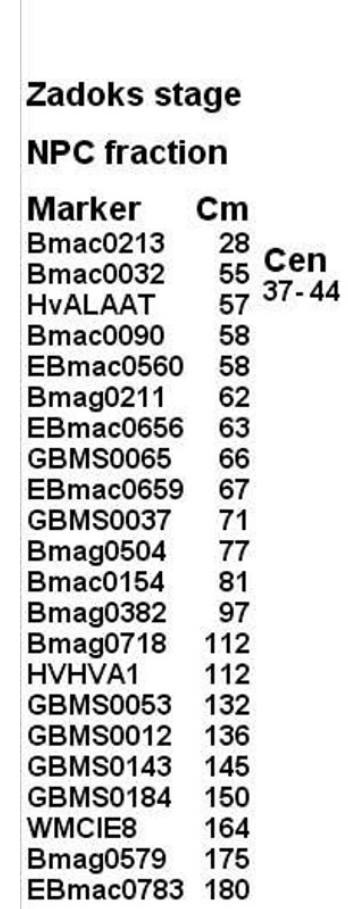

$7 \mathrm{H}$

A

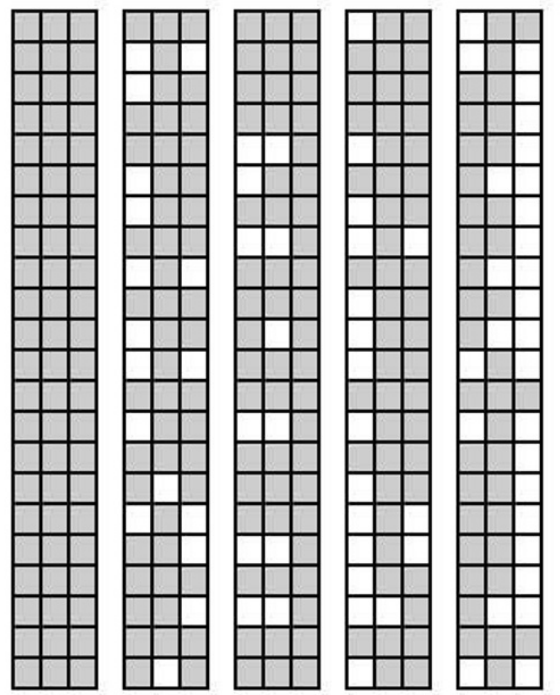

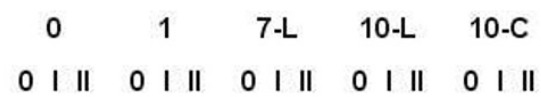

B

$$
\begin{array}{ll}
\text { Dynamics Involvement } \\
\text { in NPC }
\end{array}
$$

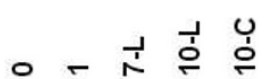

$1 \mathrm{H}$
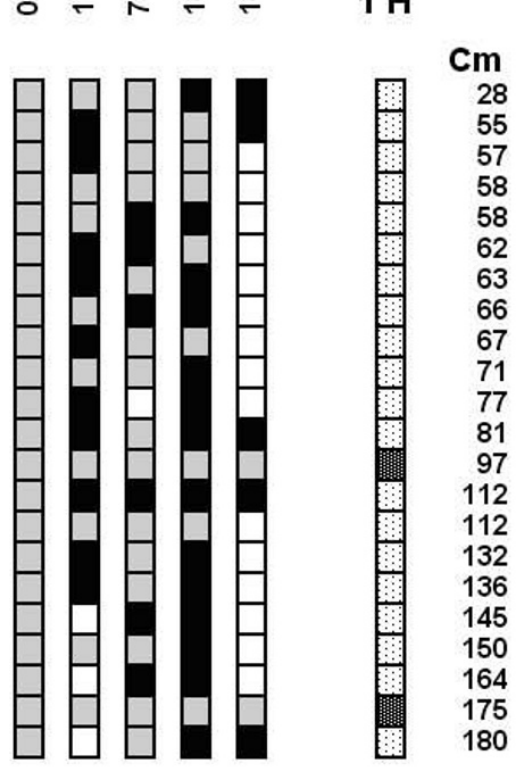

B Dynamics

C

PCR results

Zadoks stage

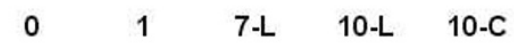
in NPC

Involvement in dynamics

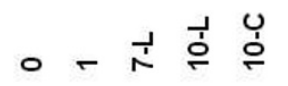

$7 \mathrm{H}$

Marker

0 । || 0 । || 0 । || 0 । || 0 । ||

$\begin{array}{lr}\text { Marker } & \text { Cm } \\ \text { GBMS0192 } & 4 \\ \text { HVPLASCIB } & 8 \\ \text { Bmag0206 } & 19 \\ \text { GBMS0139 } & 25 \\ \text { Bmag0007 } & 27 \\ \text { cMWG 773 } & 47 \\ \text { GBMS0035 } & 49 \\ \text { EBmac0603 } & 50 \\ \text { AF022725A } & 59 \\ \text { Bmag0516 } & 73 \\ \text { Bmag0914 } & 73 \\ \text { Bmac0187 } & 89 \\ \text { Bmac0031 } & 93 \\ \text { ABC 156D } & 93 \\ \text { GBMS0111 } & 100 \\ \text { Bmag0341 } & 123-128 \\ \text { Bmag0507 } & 111 \\ \text { Bmag0120 } & 118 \\ \text { cMWG 725 } & 123 \\ \text { EBmac0755 } & 145 \\ \text { cMWG 728 } & 158 \\ \text { GBMS0183 } & 161 \\ \text { Bmag0135 } & 165 \\ \text { ABG 461 } & 177\end{array}$
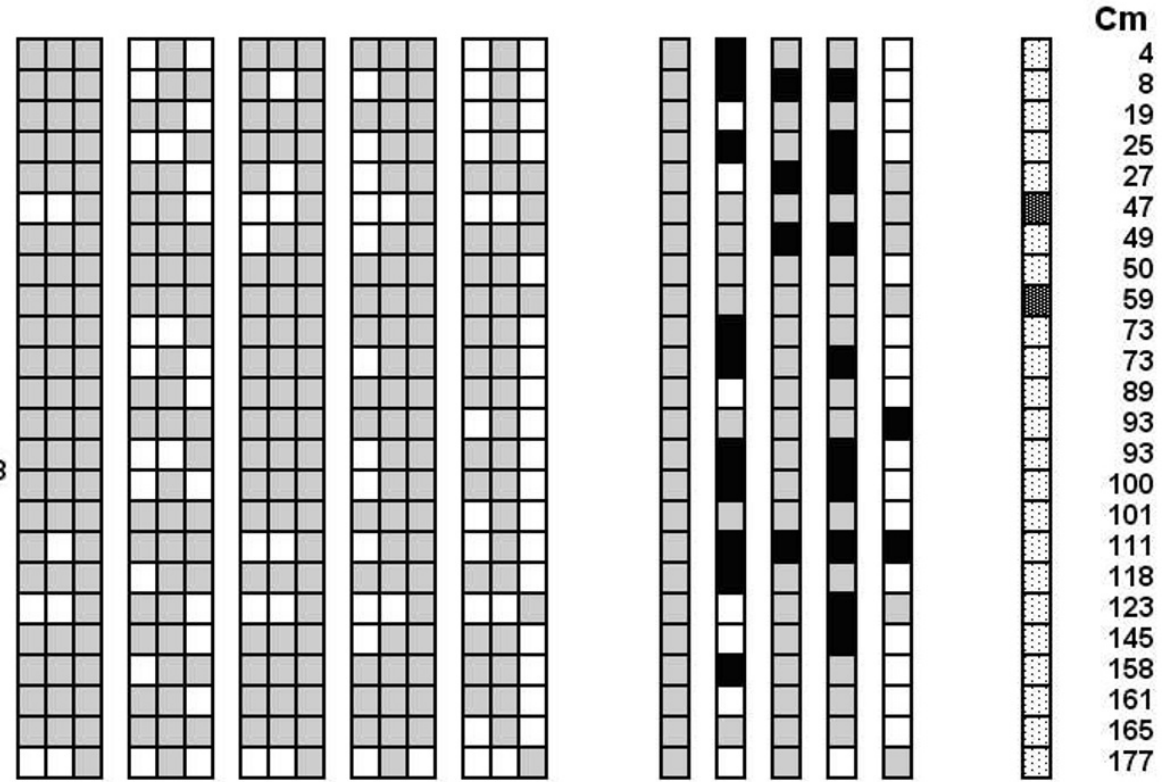

Figure 6 (see legend on next page) 
Figure 6 (see previous page)

The distribution of microsatellite sequences in fractions obtained in the course of chromatography of nucleoproteins on celite. A. The distribution of DNA fragments containing given microsatellites in unbound to nuclear matrix fraction (eluted in $\mathrm{NaCl}$ gradient, DNA 0, column 0 on the Figure), loosely bound to the nuclear matrix (eluted in LiCl-urea gradient and in temperature gradient below $70^{\circ} \mathrm{C}$, DNA I (column I) and tightly bound to the nuclear matrix (eluted in temperature gradient at $90^{\circ} \mathrm{C}$, DNA II, column II). Grey and white squares indicate presence and absence of amplification correspondingly. All other designations are like in Figure 4. B. Summary of the trends of transitions during development of different organs compared with the situation in dry seed. Grey squares - situation in the seed or similar; white squares - loosening of association with the nuclear matrix; black squares -tightening of interactions. C. General summary of involvement of the studied genomic sites in association-dissociation according the NPC chromatography data. Black points on white background - sites involved in transitions; white points on black background - sites not involved in transitions.

\section{Discussion}

\section{Gene expression changes during seed development}

In the present study we have revealed developmentdependent changes of the TBP distribution in $A m y 32 b$ and Bmy1 genes during transition of the barley seed from watery-ripe to middle milk ripe stage. Changes in TBP distribution in the genes were coupled to changes in their expression. Our RT-PCR data confirm earlier published data indicating that $\beta$-amylase expression is linked to starchy endosperm development, but $\alpha$-amylase is not expressed on late seed development stages [14], moreover over-expression of $\alpha$-amylase in developing seed leads to development defects [15].

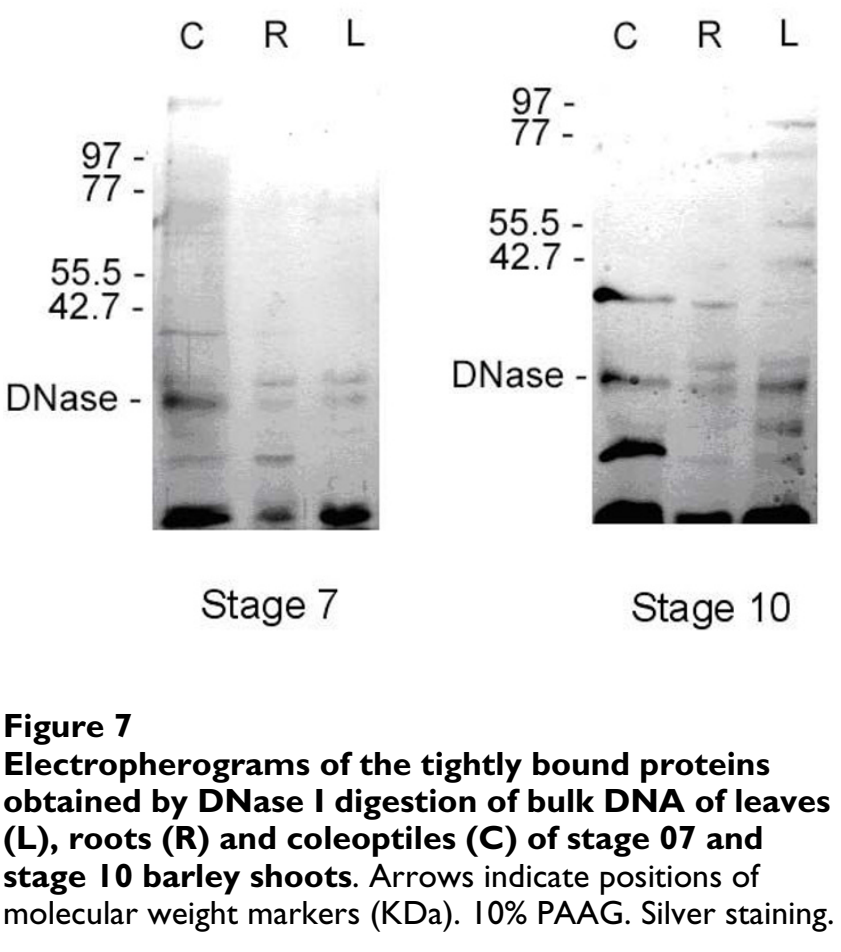

\section{TBP redistribution in the Amy32b gene}

Our observation of a decrease in TBP interactions with promoter region of the Amy32b gene is in good agreement with a recently proposed hypothesis [13]. According to this hypothesis in the dormant gene negative regulators bind to the corresponding cis-acting elements in the promoter and form a "repressosome," this diminishes the binding or transactivating activities of positive regulators to the promoter, thereby preventing Amy32b transcription. Induction of transcription is followed by binding of positive regulators to their respective DNA sequences and formation of the "enhanceosome, "leading to a high level of Amy32b gene expression. Probably, formation of the "enhanceosome" is preceded by degradation of repressors. Taking into account that the RT-PCR data expression of the gene is observed exclusively in the watery ripeness

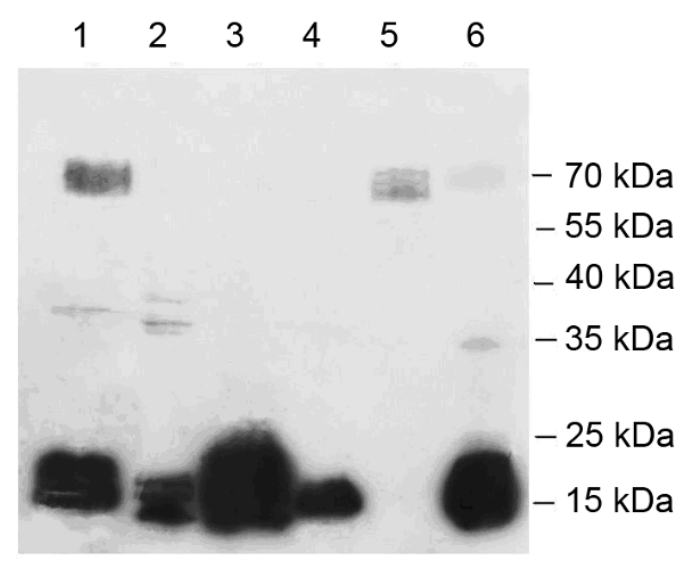

\section{Figure 8}

DNA-binding protein blot assay. Assay was performed with electrophoretically fractionated nuclear proteins isolated from the Zadoks $07(\mathrm{I}-3)$ and Zadoks $10(4-6)$ coleoptiles $(I, 4)$, leaves $(2,5)$, and roots $(3,6)$ and incubated with TBP-associated DNA from Zadoks 10 leaves. 
stage, one can speculate that TBPs could stabilize "enhanceosome", release or degradation of TBPs could be one of the mechanisms of the promoter inactivation. Interestingly in the dormant Bmy 1gene the 300 bp region upstream the translation start codon (position 4) is neither TBP-enriched. The very organization of the Amy32b gene $5^{\prime}$ area appears to be favourable for TBP binding, as it contains several inverted sequences [16], but it was shown that repeated sequences are often found in the TBP anchoring sites [3-6].

\section{$T B P$ redistribution in the $B m y /$ gene}

In Bmy 1 gene, besides the overall decrease of TBP binding coupled to up regulation of transcription we have also observed drastic changes of TBP binding in Exon 3 and Intron 3, changes $1.5 \mathrm{~Kb}$ upstream the Exon 1 were also well-pronounced. Interestingly, Intron 3 of this gene harbours a microsatellite, MITE element, several other repeats and a predicted MAR (Figure 3). Both microsatellites and mobile elements are often involved in tight DNA-protein complexes $[30,31]$ and hence these elements could also possess high affinity to TBPs. Indeed as position 10 of the microarray is very close to the microsatellite, sequences of the MITE element should hybridize with the oligonucleotide in the position 7. Enrichment of the mobile genomic elements in complexes with TBP was revealed also in our study on the characterization of the TBP-associated DNA sequences (unpublished results). On the other hand, the presence of a possible nuclear matrix attachment site (MAR) in Intron 3 of Bmy 1 gene and the absence of such sequences in other sites of the gene intensively binding TBPs indicate that TBP binding is not dependent on the presence of MAR.

\section{TBP binding and transcription}

The overall intensity of the DNA association with TBP appears to be independent of transcription, as in stage 10 seeds we observed an overall decrease in the $\mathrm{R}$ vs $\mathrm{F}$ ratio along both genes, although one of them became expressed on this stage, but the other - remained dormant. Triggering or cessation of transcription is rather accompanied by redistribution of TBPs along the gene, but not overall accumulation or release of these proteins from the whole gene sequence. The same conclusion was made after a similar study on the chicken produced alphaglobin gene domain [7]. Comparison of TBP distribution in the domain in erythroid cells differing in the degree of alpha-globin gene transcription revealed changes in distribution of TBPs, however the TBPs were much more abundant along the domain in DNA from liver than in erythrocytes and erythroblasts. Thus, in animals overall enrichment in TBPs appears to be an organ-specific trait. Our results indicate that in plants it could be characteristic not only of the organ per se, but also of the development stage of an organ.

\section{Relation between TBP and nuclear matrix attachment sites}

Application of the microsatellite analysis tool developed for the needs of marker-assisted selection during analysis of long-range DNA-protein interactions proved its utility especially for work with a partly sequenced genome like the barley genome. It should be mentioned that using the conventional PCR protocol we could observe only the most drastic changes in the sequence distribution of fractions; a quantitative real-time PCR technique would substantially increase the sensitivity of the approach. Nevertheless the assay enabled us to obtain interesting results. For example, the relation between TBP and nuclear matrix binding sites has been discussed for a long time and in some reports these are claimed to be identical structures [32-34], others consider TBP-DNA complexes to be specific structures, not related to nuclear matrix attachment sites [2,35]. Comparative study of the changes in distribution of complexes with TBPs and the nuclear matrix along Chromosomes $1 \mathrm{H}$ and $7 \mathrm{H}$ in the first leaf of the barley shoots indicates detachment of several sites in both chromosomes of the nuclear matrix during transition from watery to milky ripeness, at the same time the number of interaction with TBPs is increased (Figures 4 and 5). Thus, TBPs appear to form complexes different from the nuclear matrix association sites to DNA. Data from nucleoprotein celite chromatography are coherent with results obtained with salt-extracted nuclear matrices, but reveal the same process in more detail (Figures 5 and 6 ). The presence of a possible MAR only in one site of TBP accumulation also indicates the different nature of the two types of DNA-protein complexes.

\section{Individual peptides of TBP fraction}

Detailed characterization of the TBP spectrum by means of 2D electrophoresis and mass-spectrometry are currently ongoing. However our preliminary results on development-dependent changes in TBP spectrum reported here are in good agreement with microsatellite analysis data. Indeed, we observe some increase in the TBP-binding sites during the transition of the first leaf from stage 07 to stage 10 , the number of polypeptides in the TBP preparation increases, while in coleoptile we observed a decrease in the TBP binding sites and the peptide spectrum of TBPs is also simplified (Figures 7 and 8). Protein blot assays confirmed the same trend: in nuclear extracts of stage 07 coleoptiles several peptides bind TBP-associated DNA (Figure 8), in stage 10 coleoptiles the only lowmolecular peptide, also prominent on electropherograms (Figure 7) retains its ability to form tight complexes with DNA.

\section{Conclusion}

Our data indicate the existence of development-dependent redistribution of the TBP along Amy32b and Bmy1 and 
along studied stretches of Chromosomes $1 \mathrm{H}$ and $7 \mathrm{H}$ in barley. Sites of the TBP binding do not co-localize with the nuclear matrix attachment regions. The TBP spectrum and affinity to DNA change during plant development.

\section{Methods \\ Plant material}

Seeds of the barley cultivar Balga were obtained from the Latvian State Stende Cereal Breeding Institute and Latvian State Priekuli Crop Breeding Institute. Etiolated shoots were grown for $3-5$ days at $30^{\circ}$ in darkness. Coleoptiles, first leaves and roots were dissected from shoots of Zadoks 07 (coleoptile emerged stage, classification according [9]) and Zadoks 10 (first leaf through coleoptile) development stages. Dissected coleoptile and first leaf tissue from 50100 shoots were combined in one sample for each tissue at both developmental stages. Ten embryos per sample from dry grains (Zadoks 0 ) and after $20 \mathrm{~h}$ of imbibition (Zadoks 1) were used in some experiments. Coleoptiles, first leaf, and embryo samples were subsequently used for bulk DNA extraction. Seeds of watery ripe (Zadoks 71) and medium milk development (Zadoks 75) stages were collected in the field of the Latvian State Priekuli Crop Breeding Institute. $70-100 \mathrm{mg}$ of seed tissue was used for bulk DNA and RNA isolation.

\section{Genes}

GenBank accession X05166 sequence information was used to develop primers and the microarray and to analyze data on the barley Amy32b gene [16]. GenBank accession $\underline{\mathrm{AF} 061203}$ sequence information deposited for Adorra cultivar was used in this study for Bmy1 gene. The previously reported data [24] indicate the identity of the selected gene sequences in the Adorra and Balga cultivars used in this study.

\section{DNA and RNA isolation}

Plant tissues were frozen in liquid nitrogen and ground in a mortar or in Tissuelyser (Qiagen) to a fine powder. DNA from plant material was extracted according to the previously described protocol using chloroform-isoamyl alcohol extraction [36] with some modifications. Taking into account our previous observations on partial degradation of TBPs by proteases and the formation of artificial DNA-protein complexes due to the RNaseA treatment (reported also in [37]), we excluded the exogenous enzymes from DNA extraction protocols. RNA was precipitated with concentrated $\mathrm{LiCl}$ solution (up to $4 \mathrm{M}$ ) with subsequent $1 \mathrm{~h}$ incubation on ice [38] and removed as a pellet by centrifugation. DNA was precipitated with 1 volume of butoxyethanol. Nucleic acids from the NPC-chromatography fractions were concentrated by absorption on hydroxyapatite and elution with $1 \mathrm{ml}$ of $0.24 \mathrm{M}$ phosphate buffer. DNA was purified using a Wizard DNA Clean-Up System kit (Promega).
Total RNA was isolated according to the RNA extraction protocol of Rneasy Plant Mini Kit (Qiaqen). Samples of 2 $\mu \mathrm{g}-3 \mu \mathrm{g}$ each were stored at $-20^{\circ} \mathrm{C}$ until use.

\section{Isolation of TBP-DNA complexes by means of fractionation on nitrocellulose}

DNA was digested with HindIII and Pst1 restrictases or alternatively with AluI restrictase $(1 \mathrm{U} / 1 \mu \mathrm{g}$ of DNA) at $37^{\circ} \mathrm{C}$ overnight in appropriate buffer. For microarray experiments DNA was fragmented by sonication in a SONICS Vibra Cell ${ }^{\mathrm{TM}}$ device (Sonics \& Materials, 53 Newtown, USA) with $60 \%$ output for a period of time ranging from 30 seconds to 4 minutes. Completeness of DNA digestion or extent of fragmentation was tested by gel electrophoresis. DNA samples with average fragment sizes of about 500 bp were diluted in $3 \mathrm{ml}$ of filtration buffer $(0.5$ $\mathrm{M} \mathrm{KCl}, 5 \mathrm{mM}$ EDTA, $10 \mathrm{mM}$ Tris- $\mathrm{HCl}, \mathrm{pH} 7.4$ ) to a final concentration of $25-70 \mu \mathrm{g} / \mathrm{ml}$. The solution was pressed through a nitrocellulose filter (HAWP 02500 , HA $0.45 \mu$, $25 \mathrm{~mm}$, Millipore or NC 45, $0.45 \mu \mathrm{m}, 25 \mathrm{~mm}$, Whatman (Schleicher \& Schuell) pre-soaked with filtration buffer and supported in a Swinnex holder. The filter was washed up to five times with the same $3 \mathrm{ml}$ volume of filtration buffer to avoid contamination between fractions. Filtration buffer wash off resulted in filtered DNA fraction (F). The filter retained a DNA fraction enriched in the tightly bound proteins; it was eluted with five washes of $3 \mathrm{ml}$ each of $5 \mathrm{mM}$ EDTA, $10 \mathrm{mM}$ Tris $\mathrm{HCl}, \mathrm{pH} 7.4$ (low-ionic strength eluted fraction R1) and $3 \mathrm{ml}$ of $50 \mathrm{mM} \mathrm{NaOH}$ (alkali-eluted fraction R2) in sequence [4]. The DNA content in the F, R1 and R2 fractions was measured spectrophotometrically.

\section{Chromatin fractionation and nuclear matrix isolation}

A protocol of conventional extraction of nuclei with high salt solutions modified for monocots [39] was used for chromatin fractionation and nuclear matrix isolation. Briefly, nuclei isolated using 1\% Triton X100 extraction were consecutively extracted with:10 mM Tris $\mathrm{HCl}, \mathrm{pH}$ 7.5; $10 \mathrm{mM} \mathrm{NaCl}, 3 \mathrm{mM} \mathrm{MgCl}_{2} ; 20 \mathrm{mM} \mathrm{NH}_{4} \mathrm{Cl}$ to obtain the nucleoplasm fraction; with $10 \mathrm{mM}$ Tris $\mathrm{HCl}, \mathrm{pH}$ 7.5; $0.2 \mathrm{mM} \mathrm{MgCl}_{2}$ to obtain the fraction of soluble chromatin; and with $2 \mathrm{M} \mathrm{NaCl}, 10 \mathrm{mM}$ Tris $\mathrm{HCl}, \mathrm{pH} 7.5 ; 0.2 \mathrm{mM}$ $\mathrm{MgCl}_{2}$ to obtain the fraction of insoluble chromatin. The residual fraction contained nuclear matrix.

\section{Chromatography of nucleoproteins on celite (NPC- chromatography)}

The NPC-chromatography was performed as described $[28,29]$. Barley embryos or organs taken from 5 shoots were homogenized in a tightly-fit Dounce homogenizer in $10 \mathrm{mM}$ Tris-HCl, $\mathrm{pH} 7.6 ; 5 \mathrm{mM} \mathrm{MgCl}_{2}, 1 \%$ Triton X100. The lysate was directly applied on a precooled $\left(0^{\circ} \mathrm{C}\right)$ water-coated column of Celite R-630 (Fluka). The column was rinsed with $50 \mathrm{ml}$ of $5 \mathrm{mM} \mathrm{MgCl}_{2}, 10 \mathrm{mM}$ Tris $\mathrm{HCl}$, 
pH 7.4 (breakthrough fraction) at $0^{\circ} \mathrm{C}$ and $80 \mathrm{ml}$ of $\mathrm{NaCl}$ in linearly increasing concentration $(0 \rightarrow 2 \mathrm{M})$ and the same temperature was pumped through the column. The fraction eluted in $\mathrm{NaCl}$ gradient (DNA 0) was considered to be unbound to the nuclear matrix. Then a gradient of $\mathrm{LiCl}$ - urea $(0 \rightarrow 4 \mathrm{M} ; 8 \mathrm{M})$ was applied in the same manner. Finally the column was gradually heated from $0{ }^{\circ} \mathrm{C}$ to $100^{\circ} \mathrm{C}$ under a constant flow of $4 \mathrm{M} \mathrm{LiCl}, 8 \mathrm{M}$ urea solution. Fractions eluted in LiCl-urea gradient and at a temperature below $70^{\circ} \mathrm{C}$ were combined (DNA I) and was considered to represent a fraction loosely bound to the nuclear matrix in contrast to the DNA II fraction eluted at high temperature $\left(70^{\circ} \rightarrow 100^{\circ} \mathrm{C}\right)$ and considered to be tightly attached to the nuclear matrix.

\section{TBPs sample preparation and electrophoresis}

Protein samples were obtained using the method of exhaustive DNase digestion [6]. The DNA was digested with DnaseI (Fermentas) (1 U/100 $\mu \mathrm{g}$, room temperature, overnight) in $10 \mathrm{mM}$ Tris-HCl, $\mathrm{pH} 7.6 ; 5 \mathrm{mM} \mathrm{MgCl}_{2}$. Completeness of digestion was monitored by gel electrophoresis. Proteins were concentrated by evaporation of excess water. The DNA digest was mixed with the sample buffer, heated to $100^{\circ} \mathrm{C}$ for 5 minutes and cooled. Electrophoresis was performed in $10 \%$ or $15 \%$ PAAG gels using the conventional protocol for Laemmli system. Gels were silver stained according to [40].

\section{PCR based marker chromosome profiling}

Twenty two and twenty four primer pairs representing barley markers mapped along chromosomes $1 \mathrm{H}$ and $7 \mathrm{H}$ correspondingly were chosen for analysis (Figures 5, 6, 7). The primer sequences, genetic map position and microsatellite motif of HvALAAT, HVHVAI, Bmac, EBmac, Bmag and EBmag markers were previously published [24] and are publicly available http://bioinf.scri.ac.uk/ssr/ barley s.html. GBMS markers were developed and mapped in IPK [26]. The complete sequences of GBMS as well as WMCIE8, cMWG 773, AF022725A, cMWG 725, cMWG 728, ABG 461 markers are available from IPK on request. Markers of $\mathrm{CMWG}, \mathrm{ABC}$, and $\mathrm{ABG}$ series are primers of sequenced genomic RFLP clones [27], other markers are microsatellites.

To equalize the DNA content in different samples after fractionation on nitrocellulose, an aliquot of the $\mathrm{F}$ fraction was diluted 5 times, and aliquots of the R1 and R2 fractions were diluted twice. $5 \mu \mathrm{l}$ of the diluted solution was taken for the amplification reaction. The final PCR reaction mix contained: about 100 ng of genomic DNA; $1.5 \mathrm{mM} \mathrm{MgCl}{ }_{2}, 40 \mu \mathrm{M}$ of dCTP, dGTP, dTTP, dATP; 0.4 $\mu \mathrm{M}$ of each primer; and $1 \mathrm{U}$ of Taq polymerase in a $25 \mu \mathrm{l}$ reaction mixture. The PCR conditions were: $3 \mathrm{~min}$ at $94^{\circ} \mathrm{C}$, followed by 45 cycles at $94^{\circ} \mathrm{C}$ for $1 \mathrm{~min}, 60^{\circ} \mathrm{C}$ for $1 \mathrm{~min}$, and $72^{\circ} \mathrm{C}$ for $2 \mathrm{~min}$ with chain elongation of $7 \mathrm{~min}$
[26]. PCR was performed on a Perkin Elmer PCR machine in 96-well format. Fragment length analysis was performed on an ALF II Express automated laser fluorescence sequencer (Pharmacia) using a short gel cassette. Denaturing polyacrylamide gels were prepared following the manufacturer's protocol (Pharmacia). The sample standard mix was heat denatured and snap cooled before loading. The gels were run in $0.5 \times$ TBE buffer with $600 \mathrm{~V}, 50 \mathrm{~mA}$ and $50 \mathrm{~W}$ and gel temperature $50^{\circ} \mathrm{C}$. Fragment sizes were calculated using the Fragment Analyser 1.02 computer program (Pharmacia) via comparison with the internal size standards (73, 122, 196, and 231 bp). Results were scored as presence/absence of amplification.

\section{DNA-binding protein blot assay}

The assay was performed according to [41]. Nuclear proteins were electrophoretically separated in one dimensional 12\% SDS-polyacrylamide gel. Proteins were electroblotted from the gel onto $0.45 \mu \mathrm{m}$ nitrocellulose filters (Bio-Rad) in $19 \mathrm{mM}$ glycine, $25 \mathrm{mM}$ Tris, $0.1 \%$ SDS, and $20 \%$ methanol. Protein blots were blocked overnight at room temperature in blocking buffer $(10 \mathrm{mM}$ Tris- $\mathrm{HCl}$, pH 7.5, $50 \mathrm{mM} \mathrm{NaCl}, 2 \mathrm{mM}$ EDTA, $1 \%$ blocking reagent (BSA) and subsequently preincubated for 1 hour in binding buffer (10 mM Tris-HCI pH 7.4, $50 \mathrm{mM} \mathrm{NaCl}, 2 \mathrm{mM}$ EDTA, $0.05 \%$ BSA). Later, the membranes were transferred into a fresh portion of the binding buffer supplemented with $10 \mathrm{ng} / \mathrm{ml}$ of radioactively labelled DNA probe $(10,000-70,000 \mathrm{cpm} / \mathrm{ml})$ and $100 \mathrm{ng} / \mathrm{ml}$ of competitor DNA (Eco R1 digested plasmid pUC19). TBPbound DNA fragments obtained according to the exhaustive DNaseI digestion procedure were used as probes. Binding was carried out overnight at $37^{\circ} \mathrm{C}$ in a hybridization oven under gentle agitation. The membranes were washed three times for $15 \mathrm{~min}$ in $100 \mathrm{ml}$ of binding buffer and autoradiographed.

\section{DNA probe labelling}

Different DNA fractions were labelled with $\left[\alpha^{32} \mathrm{P}\right] \mathrm{dATP}$ (Hartmann Analytic, Germany) using a HexaLabel ${ }^{\mathrm{TM}}$ DNA Labeling Kit (UAB Fermentas, Lithuania). Unincorporated nucleotides were removed by selective precipitation of DNA with ethanol in the presence of ammonium acetate.

\section{DNA array}

Amy32b (GenBank accession ․05166) and Bmy1 (GenBank accession $\underline{\mathrm{AF} 061203}$ ) genomic sequence information was used to design the arrays. The DNA array for each gene was developed according to the published approach $[7,42]$, and consisted of 50-60 oligonucleotides spaced along the whole gene. Sequences of the oligonucleotides are given in Table 1 and Table 2, their location in the structural genes is shown in Figures 2 and 3, for $A m y 32 b$ and $B m y 1$ genes, respectively. The oligonucleotides had a similar $\mathrm{T}_{\mathrm{m}}$. Prior to hybridization the oligonucleotides 
Table I: Amy32b array oligonucleotide sequences.

\begin{tabular}{cl}
\hline Name & Sequence \\
\hline 1 & 5'-CCTCCCACGTTTATCTTCAATTTGTCAAAAAAATCATGTTCGGACCGTT-3' \\
2 & 5'-CAAAAGGTATATCCTGCGTAATATTTCTGTTACTGCACCACATTAAGAACAGTTTATATG-3' \\
3 & 5'-CAACGCTGGGTGATCCCAGCTTGGATAGTGCTATCTTTTCCCATGGAATTTGTGCCGGCC-3' \\
4 & 5'-TATCCATGCAGTGCCTCCAAGCAACACTCCACGGGGACGTAGCTCGTGTT-3' \\
5 & 5'-CAGTCTTGTGAATCATTCATCCACAGAACAAGAGTGCAGCGAACAGTGTAGATC G-3' \\
6 & 5'-CAACGAAGGTCCCTCTTCACACTAAAATCATTCGTGTCTCAACTGAACATC-3' \\
7 & 5'-AACGTGCAAATACGATCAAACAAGTATACAGTATACTGTACAAACTAAAAC-3' \\
8 & 5'-GACCACCTCAACGACCGCGTCCAGCGCGAGCTCAAGGAGTGGCTCCTCTGGCTCAAGAGC-3' \\
9 & 5'-CATGTGGCCATTCCCCTCCGACAAGGTCATGCAAGGCTACGCATACATCC-3' \\
I0 & 5'-AATCATTCAGGAAACTAAAAATCTCTTGTCTTGTCGGTTTGCAGTTCTACGACCATTTCTTT-3' \\
II & 5'-AAGCCTCCACTCATCCACCATTCAATCGAGCATGCATGAATTTTCCAAAATAATG-3' \\
\hline
\end{tabular}

were analyzed in silico to avoid repetitive DNA sequences. The oligonucleotides were slot-blotted onto membrane Hybond Zeta Probe GT +0.4 M NaOH filters in $10 \times$ SSC and fixed by baking at $80^{\circ} \mathrm{C}$ for $2 \mathrm{~h}$. The hybridization was carried out at $65^{\circ} \mathrm{C}$ in modified Church buffer $(0.5 \mathrm{M}$ phosphate buffer, pH 7.2, 7\% SDS, 10 mM EDTA) overnight. The blot was washed subsequently in $2 \times$ SSC, $0.1 \%$ SDS twice for $30 \mathrm{~min}$, then in $1 \times$ SSC, $0.1 \%$ SDS for 30 min. The blots were exposed to Phosphorimager Fuji FLA5100 for 3-12 h. The average of three independent experiments (two hybridizations per experiment) is presented. The signal of the total DNA hybridization was subtracted from the $\mathrm{F}$ and $\mathrm{R}$ fraction hybridization signal. Data are presented as $\mathrm{R} / \mathrm{F}$ ratio of hybridization signals.

\section{RT-PCR}

RNA samples of $2-3 \mu \mathrm{g}$ were pretreated with DNaseI using DNA-free kit (Ambion) and used for cDNA synthesis with Revert $\mathrm{Aid}^{\mathrm{TM}} \mathrm{H}$ Minus First Strand cDNA Synthesis Kit (Fermentas) with unspecific primers according to the manufacturer's protocol. Aliquots containing $100-150$ ng of the first strand cDNA were used as a template for
PCR amplification performed in $30 \mu \mathrm{l}$ of the reaction mixture with a final concentration of $1 \times$ PCR buffer (Fermentas), $3 \mathrm{mM}$ of $\mathrm{MgCl}_{2}, 40 \mu \mathrm{M}$ of each dNTP, $1 \mu \mathrm{M}$ of each forward and reverse sequence-specific primers and 0.612 U of Taq polymerase (Fermentas) per sample. Cycling parameters were as follows: denaturation/RT inactivation step at $94^{\circ} \mathrm{C}$ during 3 min was followed by 30 cycles of three steps; denaturation $45 \mathrm{sec}$ at $94^{\circ} \mathrm{C}$, annealing $45 \mathrm{sec}$ at $60^{\circ} \mathrm{C}$, and extension $1 \mathrm{~min}$ at $72^{\circ} \mathrm{C}$, and final extension $5 \mathrm{~min}$ at $72^{\circ} \mathrm{C}$. Primer design was performed by the Primer 3.0 program using coding regions of the genes. Bmy1 cDNA fragment of $178 \mathrm{bp}$ in size was amplified with forward 5'-TGCCGTCCAGATGTATGC-3' and reverse 5'GCAGATGAATTCTCCGATGC-3' primers to encompass part of exon II, the whole of exon III and part of exon IV. Amy32b cDNA fragment of 447 bp in size was amplified with forward 5'-TAAGCCCAACTACGATCAGGA-3' and reverse 5'-ACGTAGGCGTCTCCTTCGTG-3' primers to encompass partly exon III and exon IV. Sequences of primers for the alpha tubulin $(T u b)$ reference gene, a ubiquitous and stably expressed gene in barley, have been pub-

Table 2: Bmyl array oligonucleotide sequences.

\begin{tabular}{cl}
\hline Name & Sequence \\
\hline 1 & 5'-ACGTTTGAACATTAACGTGTGTTTTTGGTGAAAGTGAAAAAATAGTTGAC-3' \\
2 & 5'-ATAATTGGTGAGGCACATTCTCATTTGATTGGTTAGTTTAACTTCCTTGTCACATTATTT-3' \\
3 & 5'-AAAAAAAACAAATTTAGGATGATATTTTGGGGTAACTTTTGGTGTTCAATTTGTTTTTTT-3' \\
4 & 5'-ATGTGTGCGTCTTCACTTCGTATAGGGTGCCGTTTGGTTGAGAGTTGAGA-3' \\
5 & 5'-ACTATTTCAAGGATCTAGTGCACACATATACATTATTGTTGTACATATAACATTGATACT-3' \\
6 & 5'-CACCACTCTAGTTCTCTGATGCATATTTATATAGAAGTTCAAGATGACACCAAATACAAGC-3' \\
7 & 5'-TGGTGTTATCGTCGACATTGAAGTGGGACTTGGCCCAGCTGGAGAGATGAGGTACCCATC-3' \\
8 & 5'-AGAAATATATAGGATTCATCTGTGCAACTTAAATACTTAAAATGATTTTT-3' \\
9 & 5'-TTAAATTTTTAAATTGAGTGTCTTGGGTCTTGAATTTAAGACCTTTTGACTCGGATACCA-3' \\
10 & 5'-CTATGCATTTATACTTCAACAATAAGAATAGTGAGGTAGCAC-3' \\
11 & 5'-AACAACAAAAATACACAAAAC TATCCAGGCTAAGGGAACTCGCATTGCTTA-3' \\
I2 & 5'-ATTTGTTGATTTGCAGGTGCCTATTATATACTAATAATTTAATTTTATTGTTTTCAGCCT-3' \\
I3 & 5'-AGGCTGAAGGCCCCACCTGTGGCATGGGTGGGCAAGTTAAAGGCCCTACT-3'
\end{tabular}


lished [43]. Amplification resulted in the 248 bp product of expected size.

\section{Data management and analysis}

Numbering of any loci in the genes is given from the first ATG of the corresponding genomic sequence. Identification of transcription factor binding sites and clusters, analysis of similarities between the two genes and prediction of nuclear matrix attachment regions (MAR) was performed by Genomatix software (DiAlign TF, Release 3.1, and MatInspector, Release 7.4, Smartest Release 2.2) tools at http://www.genomatix.de/).

\section{Abbreviations}

TBP: tightly bound protein; TBPs: tightly bound proteins; NM: nuclear matrix; SC: soluble chromatin; IS: insoluble chromatin; MS: microsatellite; NPC-chromatography: nucleoprotein-celite chromatography.

\section{Authors' contributions}

TS initiated, coordinated, and led the project, performed PCR-based marker and bionformatical analysis and edited the manuscript. NS designed the study, analyzed the results of all the experimental work described and drafted the manuscript. MR worked out the strategy of marker analysis. YV conceived and designed the microarray experiments performed by KB. OS performed RT PCR experiments. LB, DL, KB performed experiments with proteins designed by BJ. All the authors read and approved the final manuscript.

\section{Acknowledgements}

The work was funded in part by the grant No 05. I40 I (T. Sjakste) from the Latvian Council of Science, the Deutsche Forschungsgemeinschaft grant 436 LET 17///05, and the Lithuanian State Science and Studies Foundation grant No T-109/07. Collaboration between French, Latvian and Lithuanian teams was supported by ECO-NET program 2006-2008, project "Development of an integrated genomic DNA-array based approach for the epigenetic study of the genome". Network between Latvian and Lithuanian colleagues was also supported by NordPlus Neigbour 2007 - 2008 project "Genomics for all: bringing new tools to breeders" and NordPlus Horizontal 2008 - 201 I project "Implantation of genomics and bioinformatics in agricultural practice". We thank Ina Belicka, Mara Bleidere (Latvian State Stende Cereal Breeding Institute) and Linda Legzdina (Latvian State Priekuli Crop Breeding Institute) for providing plant material.

\section{References}

I. Steinmüller K, Batschauer A, Apel K: Tissue-specific and lightdependent changes of chromatin organization in barley (Hordeum vulgare). Eur J Biochem 1986, 158:5 19-525.

2. Tsanev R, Avramova Z: Stably-bound chromosomal proteins. Chromosoma 1994, 103:293-301.

3. Pfütz M, Gileadi O, Werner D: Identification of human satellite DNA sequences associated with chemically resistant nonhistone polypeptide adducts. Chromosoma 1992, I 0 I:607-617.

4. Werner D, Neuer-Nitsche B: Site-specific location of covalent DNA-polypeptide complexes in the chicken genome. Nucleic Acids Res 1989, 17:6005-6015.

5. Neuer-Nitsche B, Lu X, Werner D: Functional role of a highly repetitive DNA sequences to anchorage of the mouse genome. Nucleic Acids Res 1988, 16:8351-8360.
6. Avramova Z, Georgiev O, Tsanev R: DNA sequences tightly bound to proteins in mouse chromatin: identification of murine MER sequences. DNA Cell Biol 1994, I3:539-548.

7. Bielskiene K, Bagdoniene L, Juodka B, Lipinski M, Sjakste T, Vassetzky YS, Sjakste N: Transcription- and apoptosis-dependent longrange distribution of tight DNA-protein complexes in the chicken alpha-globin gene. DNA Cell Biol 2008, 27:6I5-62I.

8. Sjakste T, Röder M, Labeikyte D, Bagdoniene L, Levina A, Juodka B, Sjakste N: Distribution of tight DNA-protein complexes along the barley chromosome IH, as revealed by microsatellite marker analysis. Cell Mol Biol Lett 2005, 10:49-59.

9. Anderson PM, Oelke EA, Simmons SR: Growth and Development Guide for Spring Barley. [http://www.extension.umn.edu/distri bution/cropsystems/DC2548.html].

10. Kirnos MD, Volkova SA, Ganicheva NI, Kudriashova IB, Vaniushin BF: Synchronous synthesis of DNA in coleoptiles and the initial leaf of developing etiolated wheat shoots: the nature and correlation of nuclear and mitochondrial DNA syntheses. Biokhimiia 1983, 48: I587-I595. (Russian).

II. Sjakste TG, Taurite Z, Rashal ID: Dissociation of chromatin domains during the cell cycle in developing barley seedlings. Biochemistry - Engl Tr 1993, 58:1260-1267.

12. Sjakste TG, Taurite Z, Rashal ID: Kinetin-induced changes in DNA association with nuclear matrix proteins in the first leaves of etiolated barley seedlings. Soviet Plant Physiology 1993, 40: $151-155$.

13. Zou X, Neuman D, Shen QJ: Interactions of two transcriptional repressors and two transcriptional activators in modulating gibberellin signaling in aleurone cells. Plant Physiol 2008, 148: $176-186$.

14. Mundy J, Hejgaard J, Hansen A, Hallgren L, Jorgensen KG, Munck L: Differential synthesis in vitro of barley aleurone and starchy endosperm proteins. Plant Physiol 1986, 81:630-636.

15. Green L, Faergestad EM, Poole A, Chandler P: Grain development mutants in barley. $\alpha$-Amylase production during grain maturation and its relation to endogenous gibberelic acid content. Plant Physiol 1997, I | 4:203-212.

16. Whittier RF, Dean DA, Rogers JC: Nucleotide sequence analysis of $\alpha$-amylase and thiol protease genes that are hormonally regulated in barley aleurone cells. Nucleic Acids Res 1987, 15:25I5-2535.

17. Polakova KM, Kucera L, Laurie DA, Vaculova K, Ovesna J: Coding region single nucleotide polymorphism in the barley low-pl, alpha-amylase gene Amy32b. Theor Appl Genet 2005, I 1 0:1499-1504.

18. Thacker SP, Ramamurthy V, Kothari RM: Characterization of barley $\beta$-amylase for application in maltose production. Starch 1992, 44:339-341.

19. Yoshigi N, Okada Y, Sahara H, Tamaki T: A structural gene encoding $\beta$-amylase of barley. Biosci Biotechnol Biochem 1995, 59(10): $|99|-1993$.

20. Kreis M, Williamson M, Buxton B, Pywell J, Heigaard J, Svendsen I: Primary structure and differential expression of $\beta$-amylase in normal and mutant barleys. Eur J Biochem 1987, 169:5 17-525.

21. Erkkilä MJ, Ahokas H: Special barley $\beta$-amylase allele in a Finnish landrace line HA52 with high grain enzyme activity. Hereditas 200I, I34:91-95.

22. Erkkilä MJ, Leah R, Ahokas H, Cameron-Mills V: Allele-dependent barley grain $\beta$-amylase activity. Plant Physiol 1998, I I 7:679-685.

23. Sjakste T, Röder M: Distribution and inheritance of beta-amylase alleles in North European barley varieties. Hereditas 2004, 141:39-45.

24. Sjakste TG, Zhuk AF: Novel haplotype description and structural background of the eventual functional significance of the barley $\beta$-amylase gene intron III rearrangements. Theor Appl Genet 2006, I I 3:1063-1079.

25. Ramsay L, Macaulay M, degli Ivanissivich S, MacLean K, Cardle L, Fuller J, Edwards K, Tuvesson S, Morgante M, Massari A, Maesti E, Marmiroli N, Sjakste T, Ganal M, Powell W, Waugh R: A simple sequence repeat-based linkage map of barley. Genetics 2000, 156:1997-2005

26. Li JZ, Sjakste TG, Röder MS, Ganal MW: Development and genetic mapping of 127 new microsatellite markers in barley. Theor Appl Genet 2003, 107:1021-1027.

27. Künzel G, Korzun L, Meister A: Cytologically integrated physical restriction fragment length polymorphism maps for the bar- 
ley genome based on translocation breakpoints. Genetics 2000, I54:397-4I2.

28. Lichtenstein AV, Zaboykin MM, Sjakste NI, Alechina RP: Differential dissociation of chromatin digests: a novel approach revealing a hierarchy of DNA-protein interactions within chromatin domains. J Cell Sci 1991, 99:503-5I3.

29. Sjakste $\mathrm{N}$, Sjakste T: Possible involvement of DNA strand breaks in regulation of cell differentiation. Eur J Histochem 2007, 5 I:8I-94.

30. Boulikas T: Chromatin domains and prediction of MAR sequences. Int Rev Cytol 1995, 162A:279-388.

31. Nabirochkin S, Ossokina M, Heidmann T: A nuclear matrix/scaffold attachment region co-localizes with the gypsy retrotransposon insulator sequence. I Biol Chem 1998, 273:2473-2479.

32. Razin SV, Chernokhvostov VV, Vassetzky ES: The distribution of tightly bound proteins along the DNA chain reflects the type of cell differentiation. Nucleic Acids Res 1988, 16:3617-3633.

33. Neuer-Nitsche B, Werner D: Sub-set characteristics of DNA sequences involved in tight DNA: polypeptide complexes and their homology to nuclear matrix DNA. Biochem Biophys Res Communs 1987, 147:335-339.

34. Bagdoniene L, Bonikataite K, Borutinskaite V, Labeikyte D, Juodka B: DNA sequences involved in yeast tightly bound DNA-protein complexes. Biologija 2005: I-8.

35. Avramova Z, Tsanev R: Stable DNA-protein complexes in eukaryotic chromatin. J Mol Biol 1987, 196:437-440.

36. Plaschke J, Ganal MW, Röder MS: Detection of genetic diversity in closely related bread wheat using microsatellite markers. Theor Appl Genet 1995, 91:1001-1007.

37. Benore-Parsons M, Ayoub MA: Presence of RNase A causes aberrant DNA band shifts. Biotechniques 1997, 23:I28-I3I.

38. Manning K: Isolation of nucleic acids from plants by differential solvent precipitation. Analytical Biochemistry I99|, 195:45-50.

39. Reshetnikov VN, Nenadovich RA, Gorbatsevich VI: Changes in lipid composition of rye interphase cell nuclei as a function of genome expression. Doklady of the National Academy of Sciences of Belarus 2000, 44:56-59.

40. Blum H, Beier H, Gross JH: Improved silver staining of plant proteîns, RNA and DNA in polyacrylamide gels. Electrophoresis 1987, 8:93-99.

41. Ludérus ME, de Graaf A, Mattia E, den Blaauwen JL, Grande MA, de Jong L, van Driel R: Binding of matrix attachment regions to lamin BI. Cell 1992, 70:949-959.

42. loudinkova E, Petrov A, Razin SV, Vassetzky YS: Mapping longrange chromatin organization within the chicken alphaglobin gene domain using oligonucleotide DNA arrays. Genomics 2005, 85: |43-I5I.

43. Suprunova T, Krugman T, Distelfeld A, Fahima T, Nevo E, Korol A: Identification of novel gene (Hsdr4) involved in water-stress tolerance in wild barley. Plant Mol Biol 2007, 64:17-34.

Publish with Bio Med Central and every scientist can read your work free of charge

"BioMed Central will be the most significant development for disseminating the results of biomedical research in our lifetime. "

Sir Paul Nurse, Cancer Research UK

Your research papers will be:

- available free of charge to the entire biomedical community

- peer reviewed and published immediately upon acceptance

- cited in PubMed and archived on PubMed Central

- yours - you keep the copyright
BioMedcentral 